\title{
Effect of friction on the performance of inertial slider
}

\author{
K ANANTHESHWARA $^{1 *}, \mathrm{~N} \mathrm{~S} \mathrm{MURALI}^{2}$ and M S BOBJI ${ }^{1}$ \\ ${ }^{1}$ Department of Mechanical Engineering, Indian Institute of Science, \\ Bangalore 560012 \\ ${ }^{2}$ Space Mechanics Group, Indian Space Research Organisation, New BEL Road, \\ Bangalore 560094 \\ e-mail: anantha@mecheng.iisc.ernet.in
}

\begin{abstract}
Inertial sliders are friction based drives used to position with a resolution of a few hundred of nanometers to over a range of few millimeters. The compactness and simple construction enabled it to be used as a coarse positioner in various Scanning Probe Microscopes (SPM). Even heavy masses has been thought off earlier to use the inertial slider to position precisely, lack of understanding in the dynamical friction behaviour has been the main reason why the inertial sliders potential has not been explored fully in any practical device. In this paper, we have studied the effect of different operating parameters on the step size of the slider.

The inertial mass is kept on three sapphire balls, which are attached to shear piezoelectric material. The behaviour of inertial mass was studied for different input waveforms and different surface conditions that come in contact with the sapphire balls. It was observed that under lubricated conditions the step size was reduced.
\end{abstract}

Keywords. Inertial slider; friction; coarse positioner.

\section{Introduction}

The micro positioning over a distance of several millimeters is important in many applications. This can be achieved by anyone of the following techniques, mechanical devices in which micrometric screw activate the motion, inchworm translators, (Hosaka et al 1993) stepper motor (Wiesendanger et al 1990) and stick slip mechanism (Blackford 1993; Goken 1994; Howald et al 1992; Renner et al 1990; Libioulle et al 1993) in which piezoelectric effect is the source of deformation. Among these attractive classes is the stick slip mechanism based on inertial stick slip motion as described by Pohl (1987). Inertial slider uses a piezoelectric actuator to produce alternating periods of sliding and sticking between actuator and slider to generate steps during the motion and the step size is controlled by input waveforms. The input waveform consists of two parts, a ramp during which the voltage is increased gradually followed by a sudden drop to zero voltage. During the gradual increase the mass follows the piezoelectric movement because of friction (stick), where as it cannot follow a sudden piezoelectric retraction because of its inertia (slip). A gradual deformation followed

${ }^{*}$ For correspondence 
by abrupt backwards jump results in a relative stepping motion of the mass with respect to the piezoceramic. The compactness and simple construction enabled it to be used as a coarse positioner in various applications. Friction is an important factor in inertial sliders, since the characteristic of the surface influence on the step size of the sliders. Bobji et al (2006) referred to the coefficient of friction and the normal force as the main parameters in determining the step size.

The most interesting thing is the variation of the step size with operating parameters. It looks like for different operating conditions the ideal waveforms are different. Erlandsson \& Olsson (1996) used saw tooth signal in their micro positioning system. Woodburn et al (1993) simulated movement of the slider for linear parabolic and exponential ramps. Renner et al (1990) demonstrated cycloidal waveform for both the ramp and the drop parts of the input are more efficient than the saw tooth signal. In this paper, the inertial slider behaviours under different operating conditions and under different applied waveforms are studied.

\section{Description of experimental set-up}

To study the limits and the performances of the inertial slider under different operating conditions an experimental set-up of the inertial slider was built which will allow us to vary the different operating parameters. A shear piezo-electric plate $(5 \times 5 \times 0.75 \mathrm{~mm}$ thick $)$ is bonded between copper beryllium sheets of 0.25 thick by means of conductive epoxy. The bottom plate is rigidly fixed to a glass slide with a thin layer of cyanoacralic adhesive. Three spherical sapphire balls of $1 \mathrm{~mm}$ diameter are placed on the top plate. The balls are positioned on the top of the piezoceramic with the help of 3 holes (diameter $0.8 \mathrm{~mm}$ ) on the top $\mathrm{Cu}$-Be plate as shown in figure 1 . This restrains the ball and helps to increase the stiffness of the drive. The balls used here for defining three point contacts and hence defines a plane. A slider block is placed on the balls such that the center of gravity of the slider falls within the triangle formed by the 3 balls. Figure 1 shows a schematic of the experimental set-up developed. The electrical signal is applied to the piezoceramic through connecting wires. To create a translation motion of the slider, the piezo-electric plate is excited by an input waveform of gradual increase from 0 to $300 \mathrm{~V}$ and abrupt decrease in amplitude.

A non-contact displacement sensor is used to measure the displacement of the slider. The displacement sensor gives voltage output corresponding to the displacement of the slider. The target surface is attached to the slider. The slider is mounted on an X-Y stage that can be moved using a micro screw mechanism in both directions independently with a resolution of 10 microns. The signal is applied to the piezoelectric material and the displacement of the inertial mass is determined. The velocity of the slider is determined by measuring the
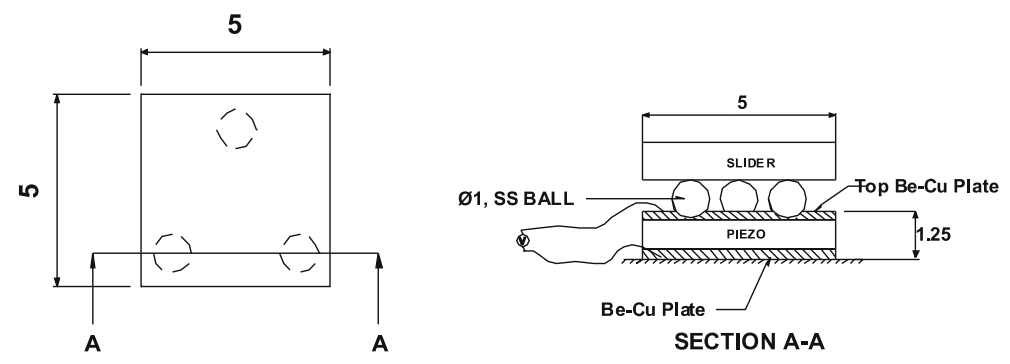

Figure 1. Schematic diagram of the piezoceramic assembly. 


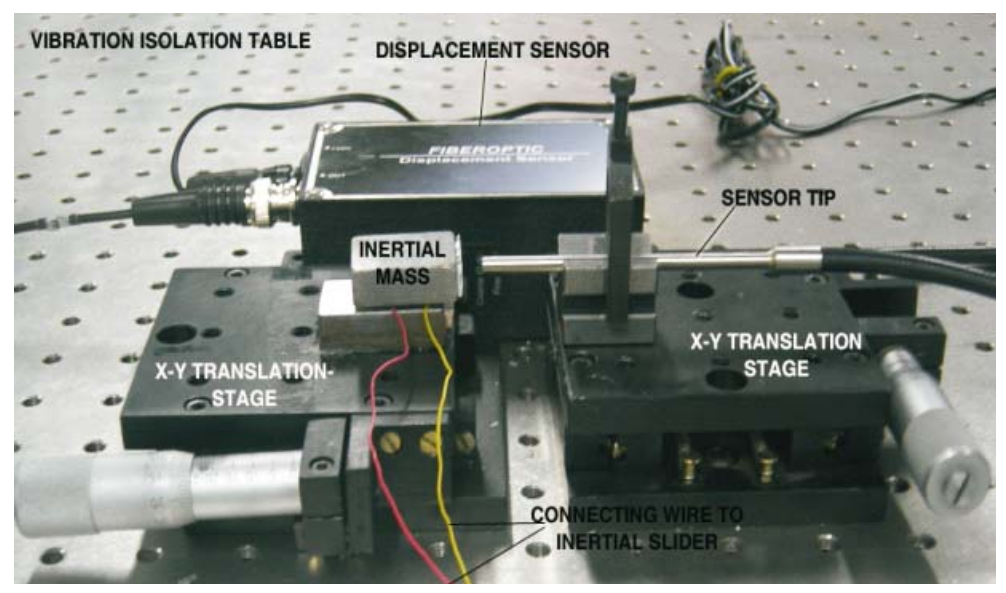

Figure 2. Experimental set-up.

displacement of the slider in $15 \mathrm{sec}$. Figure 2 shows the experimental set-up. The whole set-up is placed on a vibration isolation table to avoid any ground vibrations.

\section{Results and discussion}

\subsection{Variation of co-efficient of friction}

The frictional force is an important parameter in determining the step size. This is varied either by varying the normal load or by varying the co-efficient of friction. The different coefficient of friction is achieved by using different contact pairs (steel surface against steel balls and alumina plate against sapphire balls) and also by lubrication (vacuum grease, Shell $2 \mathrm{~T}$ ). The co-efficient of friction of these surfaces is determined using a strain gauge based force sensor. An exponential ramp with sudden drop having a frequency $121 \mathrm{~Hz}$ is applied to the piezoceramic. The average step size is determined by acquiring the displacement sensor output for $15 \mathrm{sec}$.

It is observed that as the coefficient of friction reduces the average step size reduces. The step size is highest for contact between steel surfaces with steel balls (figure 3) under dry conditions.

When signal is applied during the gradual increasing cycle (figure 4) the piezoceramic shears and the mass moves in one direction. As the input voltage drops to zero it comes back to its original position but the mass will have some finite velocity and continues to move. The mass oscillates and comes to rest after some time. Generally, the step size reduces with the reduction in the coefficient of friction under dry conditions. For a given inertial mass, the reduction in frictional force causes the slider to stick less during the slow ramp of the waveform. This results in net reduced displacement of the slider. Hence with reduction in the co-efficient of friction the average step size reduces.

\subsection{Variation of the applied ramp}

In order to understand the effect of different waveforms on the average step size the input ramp was varied. The different ramps applied to the piezoceramic are exponential, cubic quadratic, 


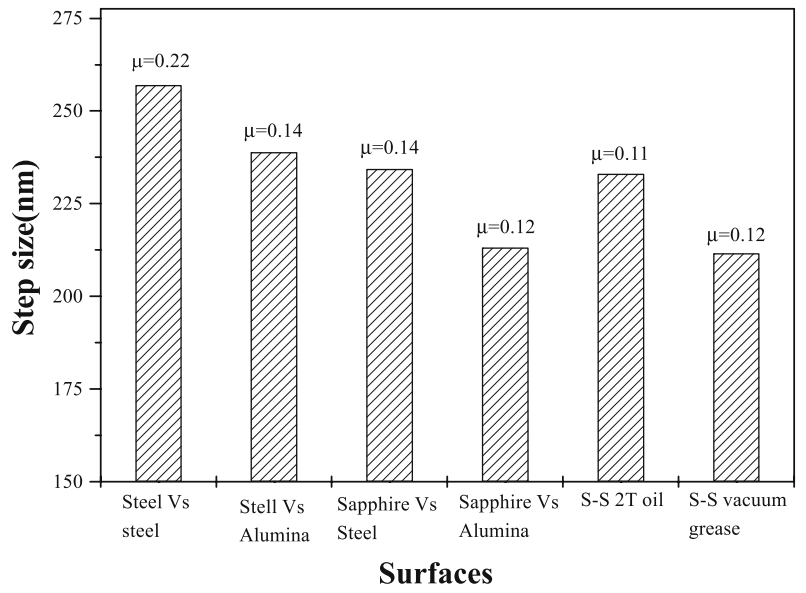

Figure 3. Variation of average step size with the different experimental conditions (mass $42 \mathrm{gm}$ ). The steel surface Vs steel balls (S-S) is studied under dry and lubricated conditions ( $2 \mathrm{~T}$ oil and vacuum grease), different contact surfaces are achieved using alumina plates and sapphire balls. The coefficient of friction is indicated at the top of the column.

linear ramps, with alumina plate Vs sapphire balls as the contact surfaces. The peak value of the input voltage for each of the waveforms is varied from $75 \mathrm{~V}$ to $300 \mathrm{~V}$. Figure 5(A) shows the different input signals applied to the piezoceramic. The variation of step size with the applied waveform is as shown in figure 5(B). It is surprising to see that for the exponential, cubic and quadratic ramps the step size is greater.

By analysing the actual displacement of the sliding mass for one period of the applied waveform (see figure 4), it was found that the main factor affecting the average step size is the velocity of the sliding mass just before the commencement of the slip. As the input voltage is suddenly dropped to zero, the inertial forces acting on the mass exceeds the friction force and the mass continues to move with the same velocity attained during the stick phase. The distance over which the mass will travel depends on this velocity and the decelerating frictional force acting on it. By varying the shape of the ramp we ensured that keeping all other conditions constant only the velocity at the end of stick phase is varied. From the figure 5 it can be seen that the step size is maximum for the exponential ramp followed by cubic, quadratic, linear ramps. To test this hypothesis further we tried an exponential ramp followed by a $1 \mathrm{~ms}$ delay during which the voltage is maintained constant. This will ensure that voltage of the

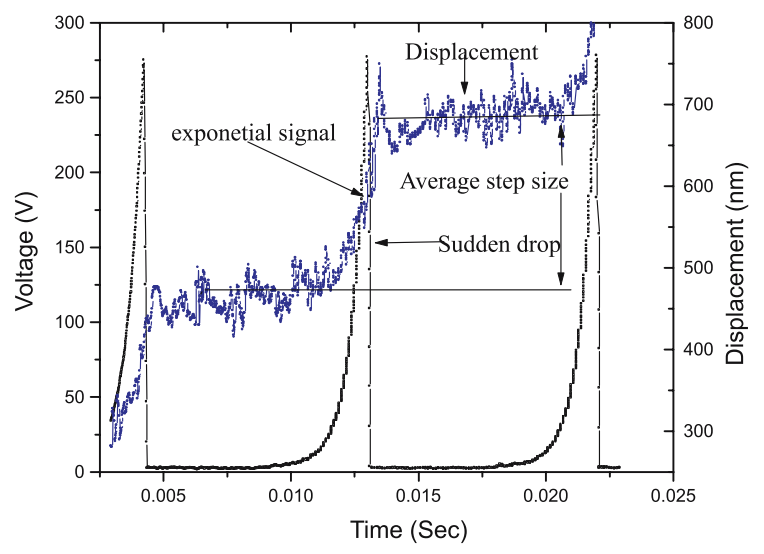

Figure 4. The motion of the inertial mass when exponential ramp is applied (mass $42 \mathrm{gm}$ ) alumina plate is in contact with the sapphire balls. 


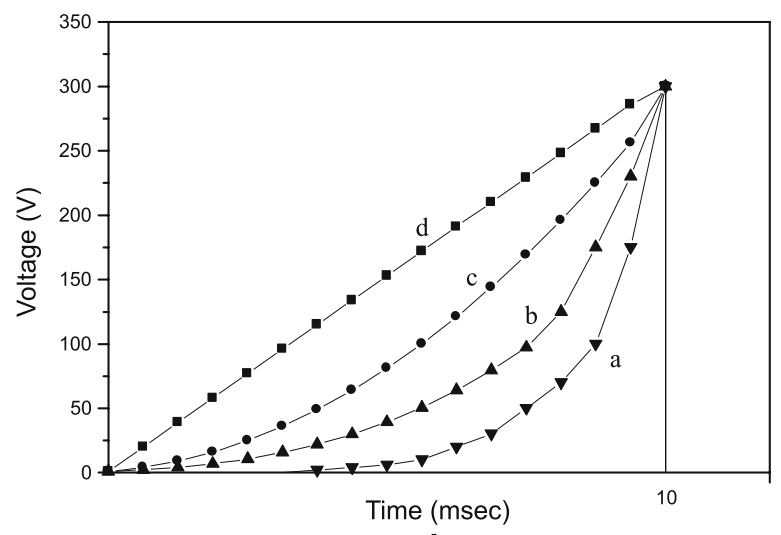

A

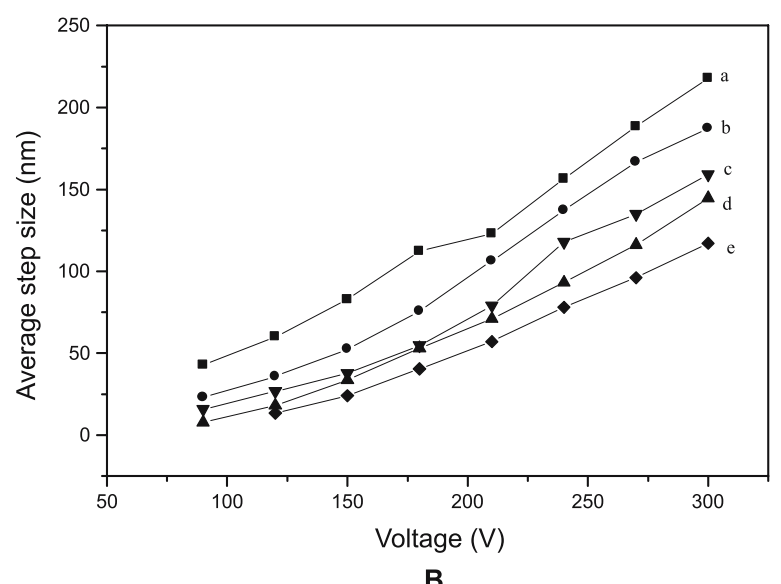

Figure 5A. Different input wave forms applied to the piezoceramic, (a) exponential, (b) cubic (c) quadratic, (d) linear increase followed by sudden drop.

Figure 5B. Variation of average step size with the applied voltage for different ramps, (a) exponential, (b) cubic, (c) quadratic, (d) linear, (e) exponential with $1 \mathrm{~ms}$ delay (mass $42 \mathrm{gm}$ ) alumina plate Vs sapphire balls as contact surfaces.

sliding mass is brought close to rest before the commencement of the sudden drop segment of the input waveform. It has been observed that this gives the lowest step size and further experiments confirmed that the shape of the input ramp before the $1 \mathrm{~ms}$ delay has very little effect on the step size.

\section{Conclusions}

The conclusions based on the experimental results are as follows:

- Coefficient of friction is one of the parameters in determining the step size, as the coefficient of friction increases the average step size increases.

- The maximum velocity of the slider is obtained when subjected to exponential waveform (ramp).

\section{References}

Blackford B L 1993 A hammer action micropositioner for scanning probe microscopes. Rev. Sci. Instrum. 64: 1360-1361 
Bobji M S, Ramanujan S, Pethica J B, Inkson B J 2006 A miniaturized TEM nanoindenter for studying material deformation insitu. Meas. Sci. Technol. 17: 1-6

Erlandsson R, Olsson L 1996 A three axis micropositioner for ultra-high vacuum use based on the inertial slider principle. Rev. Sci. Instrum. 67(4): 1472-1474

Goken M 1994 Insitu force calibration of high force constant atomic force microscope cantilevers. Rev. Sci. Instrum. 65: 2252-2254

Hosaka S, Honda Y, Hasewaga T, Yamamoto T, Kondo M 1993 Ultra high vacuum atomic force microscope using a pantograph inchworm mechanism. Rev. Sci. Instrum. 64: 3524 -3529

Howald L, Rudin H, Gunterherodt H J 1992 Piezoelectric inertial stepping motor with spherical rotor. Rev. Sci. Instrum. 63: 3909-3912

Libioulle L, Ronda A, Derycke I, Vigneron J P, Gilles J M 1993 Vertical two-dimensional piezoelectric inertial slider for STM. Rev. Sci. Instrum. 64: 1489-1494

Pohl D W 1987 Dynamic piezoelectric translation devices. Rev. Sci. Instrum. 58: 54-57

Renner Ch, Niederman Ph, Kent A D, Fischer O 1990 A vertical piezoelectric inertial slider. Rev. Sci. Instrum. 61: 965-967

Wiesendanger R, Buergler D, Tarrach G, Anselmetti D, Hidber H D, Guntherodt 1990 An ultra-high vacuum scanning tunneling microscope for the investigation of clean surfaces. J. Vac. Sci. Technol. A8: $339-344$

Woodburn N, Mckinnon A W, Roberts D A, Taylor M E, Welland M E 1993 A one-dimensional piezoelectric driven inertial micropositioner with vertical capabilities. Meas. Sci. Technol. $4535-4537$ 